\title{
Modelo numérico do terreno obtido por diferentes métodos em cartas planialtimétricas
}

\author{
$\overline{\text { Ana P. Barbosa }{ }^{1}, \text { Alessandra F. da Silva }{ }^{1} \text { \& Célia R. L. Zimback }}{ }^{1}$
}

\section{RESUMO}

O modelo numérico do terreno é importante para a determinação da declividade e da capacidade de uso das terras, motivo pelo qual foi desenvolvida uma proposta de metodologia de aquisição de dados de elevação que contemple um algoritmo eficiente para a geração de uma carta de declividades; para isto, buscou-se a obtenção e a avaliação de um modelo numérico do terreno sem a prática da vetorização das curvas de nível em cartas planialtimétricas. A área de obtenção dos dados de altitude $(\mathrm{m})$ foi no município de São Manuel, SP, onde os dados foram coletados através de dois métodos: vetorização das curvas altimétricas e o método por coleta de pontos de altitude sobre as curvas em nível com os pontos de máxima elevação. Os dados de altimetria $(\mathrm{m})$ foram analisados pela técnica geoestatística. Apesar da grande diferença no número de pontos coletados pelos dois métodos, os variogramas foram ajustados ao modelo exponencial e representaram alcance de aproximadamente $1.500 \mathrm{~m}$, o que não justifica a grande dificuldade da vetorização das cartas planialtimétricas, uma vez que os dados pontuais coletados na área foram bem distribuídos e representaram adequadamente a superfície do terreno.

Palavras-chaves: interpolação, krigagem, declividade

\section{Digital terrain model obtained by different methods in planialtimetric charts}

\begin{abstract}
The digital elevation model is important to determine the slope and land use capability, therefore, a proposal of methodology for acquisition of elevation data contemplating an efficient algorithm to generate a slope map was developed. Thus, it was aimed to obtain and evaluate a digital elevation model without the vetorization of the contours on planialtimetric charts. The area for acquisition of elevation data was São Manuel, SP. The data were collected by two methods: level contour vetorization and the gathering of elevation points on the level contour with maximum elevation points. The elevation data were analyzed by geostatistical techniques. Inspite of wide difference in the number of collected points between two methods, the variograms were adjusted to the exponential model and showed a range of approximately $1500 \mathrm{~m}$, which does not justify the wide difficulty in vetorization of the planialtimetric charts, once the data points collected in the area were appropriately distributed, they represented rightly the terrain surface.
\end{abstract}

Key words: interpolation, kriging, slope 


\section{INTRODUÇÃO}

A representação digital da superfície do terreno é fator significativo quando se buscam ferramentas auxiliares à organização espacial do ambiente.

O terreno ou topografia é um dos fatores mais importantes na agricultura influenciando as características do solo, padrões de fluxo de água, sedimentos e transporte de contaminantes, o comportamento hidrológico e métodos de irrigação. Consequentemente, a topografia afeta o rendimento das culturas, a qualidade do solo e da água e os processos de mecanização de campo (Karkee et al., 2008).

Um modelo numérico do terreno (MNT) é uma representação digital da superfície da Terra, importante para a análise quantitativa de topografia, também conhecida como análise digital do terreno ou geomorfometria (Hengl \& Reuter, 2009).

Com o início da utilização dos modelos numéricos do terreno (MNT) nos últimos anos e sua precisão cada vez maior em resolução espacial e disponibilidade, a análise quantitativa da superfície da terra tornou-se cada vez mais difundida. Segundo Hengl \& Reuter (2009) a análise geomorfométrica por MNT é amplamente aplicada em vários campos científicos, como para avaliar a probabilidade de riscos de inclinação (Guzzetti et al., 2005; Kheir et al., 2007), verificar o modelo de potencial de radiação solar (Reuter et al., 2005) na agricultura de precisão (Erdogan, 2009) entre outros.

Tipicamente, um MNT é um mapa raster (uma imagem ou um conjunto de elevação) que, como muitos outros aspectos espaciais, pode ser eficientemente modelado usando-se geoestatística (Hengl et al., 2008).

Para a geração do MNT são imprescindíveis informações altimétricas do terreno que garantam seu contexto geomorfológico. Usualmente, utilizam-se curvas em nível e pontos altimétricos como fonte de informação altimétrica porém o MNT gerado a partir de curvas em nível e pontos altimétricos como as únicas fontes de informação altimétrica, têm dois inconvenientes: o de resultar em um grande volume de dados e de apresentar inconsistências (triângulos horizontais e aresta de triângulos interceptando feições topográficas estruturais), que alteram a representação do terreno (Gonçalves \& Carvalho, 2003).

A precisão do MNT e o tamanho da grade das amostras estão relacionados intrinsecamente com a fonte de dados e o método de amostragem (Erskine et al., 2007).

A aquisição dos dados é um processo relevante na modelagem digital de elevação e deve ser realizada de modo a minimizar a quantidade de informações a serem adquiridas e que melhor representem a superfície. Os problemas existentes decorrem desta necessidade e são basicamente determinantes no melhor espaçamento da grade e na redução dos pontos das curvas de isovalor (Lourenço, 2002).

A medição manual da declividade sobre mapas com curvas em nível é um trabalho cansativo e sujeito a erros porem há disponibilidade de programas que fazem o cálculo automaticamente (Valeriano, 2003).

A metodologia geoestatística tem sido extensamente aplicada (Zhu \& Shao, 2008; Brocca et al., 2007; Cichota et al., 2006; Hébrard et al., 2006; Souza et al., 2005) e, por meio dela, é possível conhecer a continuidade de uma variável de interesse, em toda uma área de estudo, demonstrando a variação espacial da variável. Segundo Hengl et al. (2008), a geoestatística oferece vantagem significativa sobre técnicas de geração padronizada do MNT.

A krigagem ordinária é o interpolador geoestatístico mais utilizado e está disponível em vários programas computacionais. A construção de superfícies contínuas incluindo o MNT, pode ser rapidamente realizada utilizando-se a krigagem ordinária (Siska et al., 2005).

Sabendo-se da importância dos modelos de declividade para caracterização geomorfométrica do relevo, desenvolveu-se uma proposta de metodologia de aquisição de dados de elevação que contemple um algoritmo eficiente para a geração de uma carta de declividade; para isto, buscou-se a obtenção e a avaliação de um modelo de superfície do terreno sem a prática da vetorização das curvas de nível em cartas planialtimétricas.

\section{MATERIAL E MÉTODOS}

A área de obtenção dos dados de altitude (m) corresponde a um retângulo situado entre as coordenadas geográficas $22^{\circ}$ 48' 00' 54'" - 22॰ 45' 01 89" (W) e 48 36' 12,89" - 48 32' 45,76" (S), com base na carta planialtimétrica de Pratânea (IBGE, 1972), folha SF-22-Z-B-V-4, escala 1:50000, município de São Manuel, SP.

Após o georreferenciamento da carta os dados foram coletados num programa de vetorização, por dois métodos, em que no primeiro método foram coletados 750 pontos de altitude apenas sobre as curvas de nível e pontos de máxima elevação (Figura 1A); no segundo método gerou-se um arquivo de topologia linhas e pontos, por meio da vetorização das curvas de nível quando foram atribuídos, então, os valores de elevação; em seguida, as curvas foram convertidas para pontos resultando em uma malha irregular com 4708 pontos (Figura 1B).

Os dados de altimetria(m) foram analisados pela técnica geoestatística utilizada para verificar a existência e quantificar o grau de dependência espacial a partir do ajuste de funções teóricas aos modelos de variogramas experimentais, com base na pressuposição de estacionaridade da hipótese intrínseca e conforme a equação:

$$
\gamma(\mathrm{h})=\frac{1}{2 \mathrm{~N}(\mathrm{~h})} \sum_{\mathrm{i}=1}^{\mathrm{N}(\mathrm{h})}\left[\mathrm{Z}\left(\mathrm{x}_{\mathrm{i}}\right) \mathrm{Z}\left(\mathrm{x}_{\mathrm{i}}+\mathrm{h}\right)\right]^{2}
$$

em que:

$\mathrm{N}(\mathrm{h})$ - número de pares experimentais de observações $\mathrm{Z}\left(\mathrm{x}_{\mathrm{i}}\right)$, $\mathrm{Z}\left(\mathrm{x}_{\mathrm{i}}+\mathrm{h}\right)$, separados por um vetor $\mathrm{h}$

No ajuste dos modelos teóricos aos variogramas experimentais determinaram-se os coeficientes efeito pepita $\left(\mathrm{C}_{0}\right)$, patamar $\left(\mathrm{C}_{0}+\mathrm{C}\right)$ e alcance (a). Para verificar a dependência espacial utilizouse um índice de dependência espacial (IDE), proposto por Zimback (2001); para estimar valores dos dados de elevação em locais não 
A.

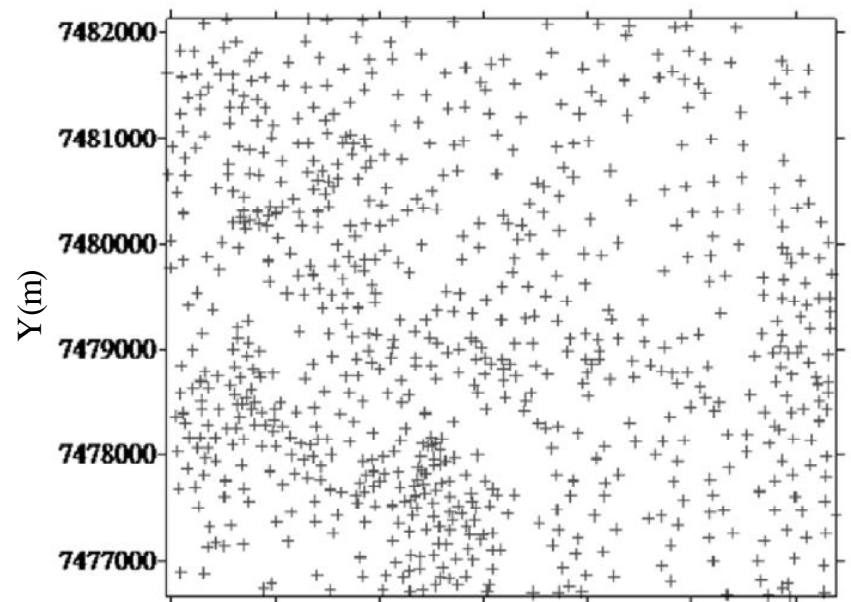

$\begin{array}{llllllll}746000 & 747000 & 748000 & 749000 & 750000 & 751000 & 752000\end{array}$
B.

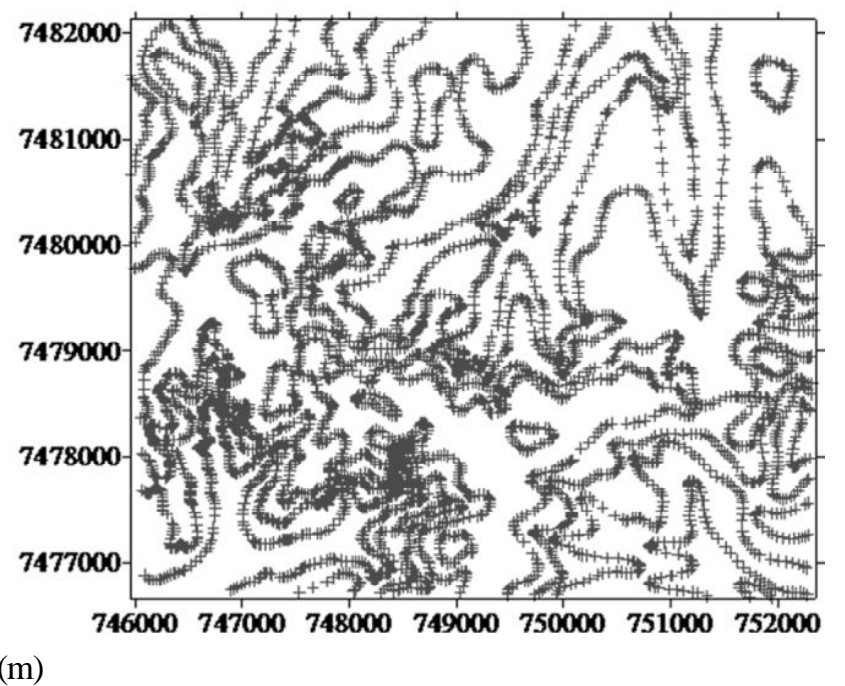

Figura 1. Mapa de amostragem dos pontos (A) e das curvas (B)

amostrados e confeccionar os mapas de isolinhas, utilizou-se a krigagem ordinária. A análise geoestatística e as interpolações foram realizadas no software $\mathrm{GS}^{+} 9.0$ (Robertson, 2008).

Visando à verificação do modelo obtido por meio da coleta de pontos na carta, calculou-se a diferença entre o mapa de krigagem das curvas e o mapa de krigagem dos pontos.

Para cada mapa de krigagem foi gerado um mapa de declividade a partir da função de variáveis topográficas utilizando-se as classes de relevo propostas pela EMBRAPA (2006).

\section{RESULTADOS E DISCUSSÃO}

Os dados de elevação pontuais e de curvas apresentaram estrutura espacial ajustando-se ao modelo exponencial (Figura 2).

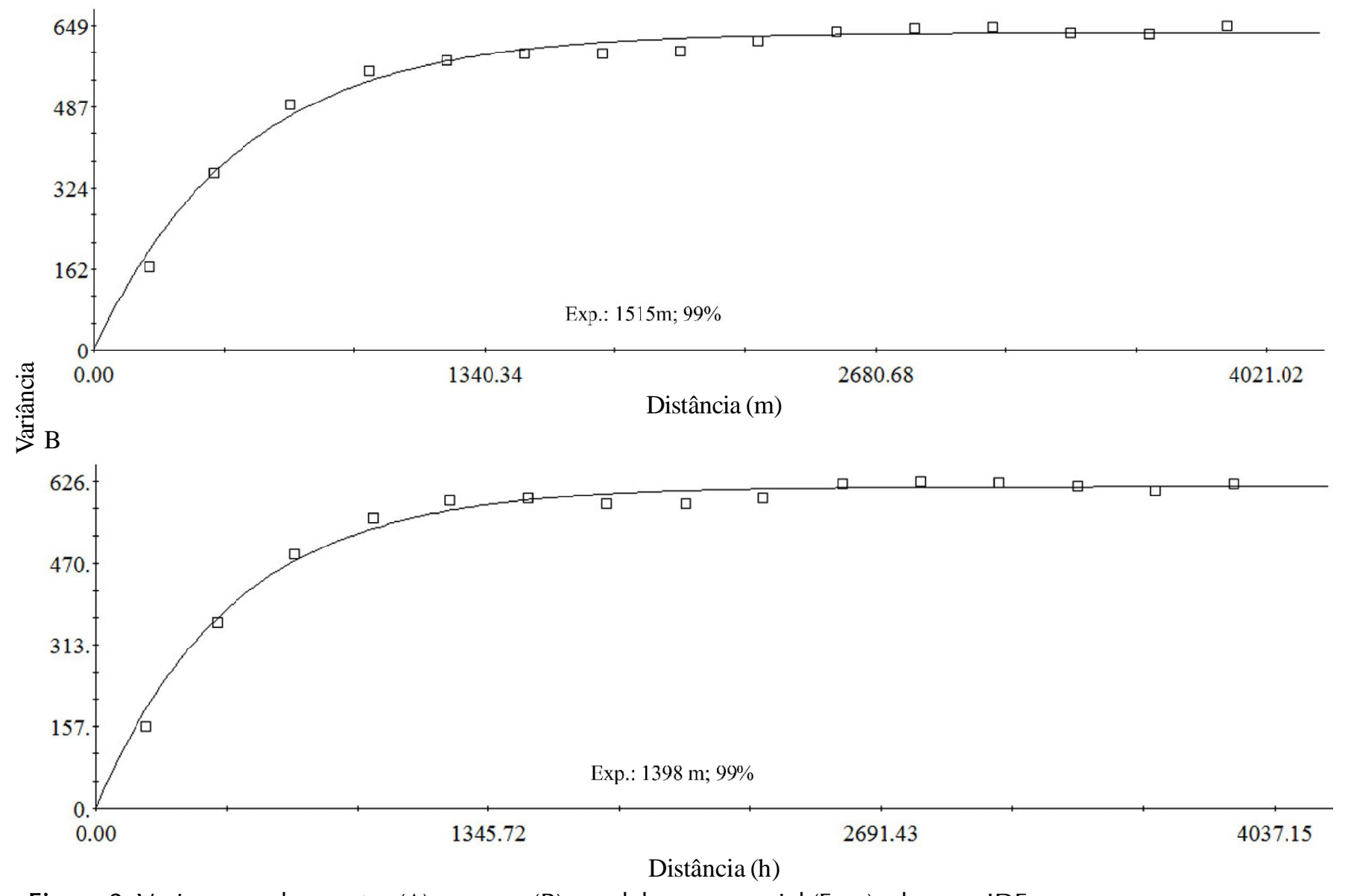

Figura 2. Variograma dos pontos (A) e curvas (B): modelo exponencial (Exp.); alcance; IDE 
Ambos os métodos de amostragem apresentaram alta dependência espacial (IDE $\geq 75 \%$ ), conforme Zimback (2001); já Valeriano (2003) encontrou fraca dependência espacial para dados de elevação.

Os métodos de amostragem tiveram valores de alcance parecidos; com isto, os mapas interpolados por krigagem apresentaram comportamento semelhante (Figura 3).

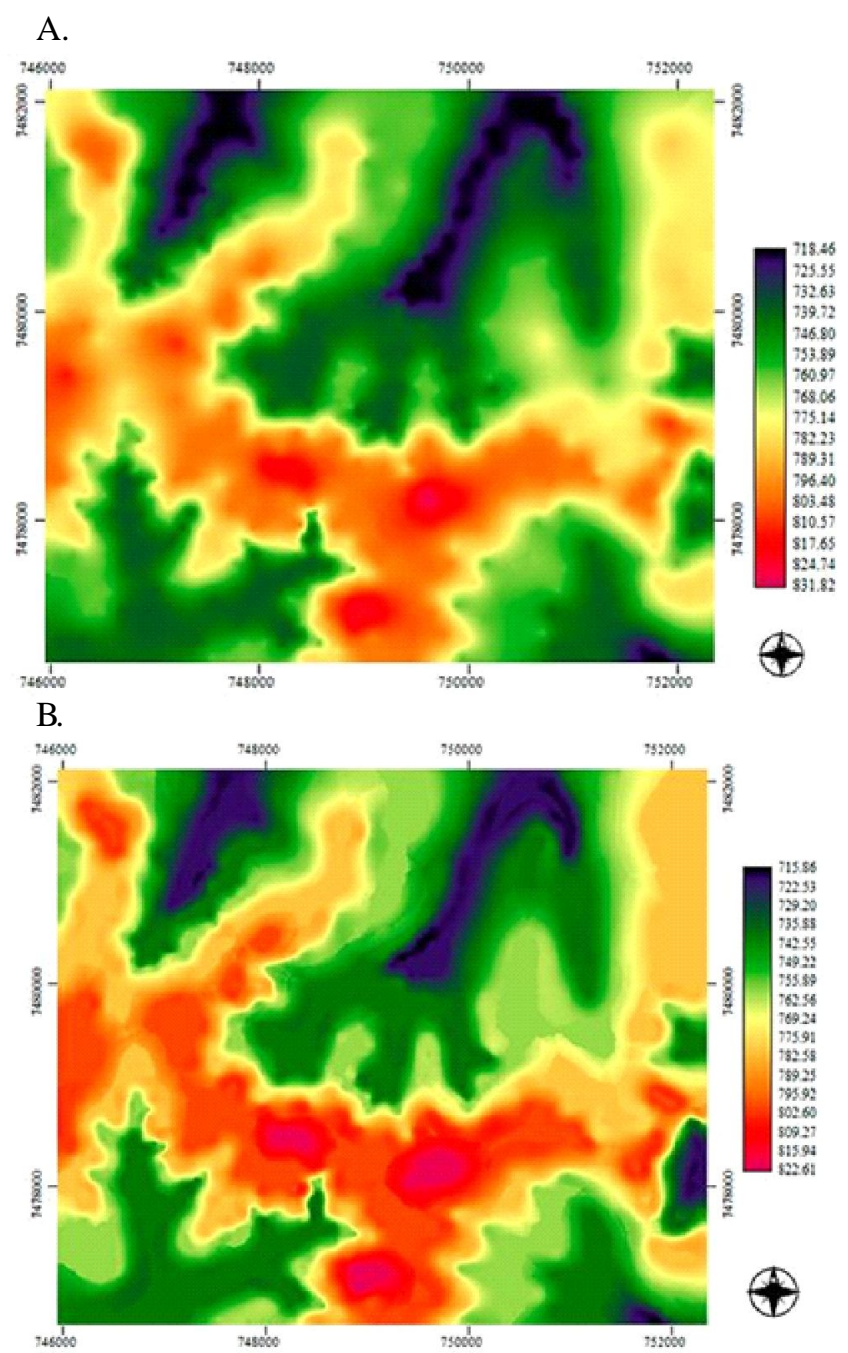

Figura 3. Mapa de krigagem dos pontos (A) e de curvas (B)

O mapeamento da declividade requer alta resolução espacial para uma representatividade adequada de sua variação, sobretudo na faixa de altos valores (Valeriano, 2003) o que se confirma nos resultados obtidos, visto que as interpolações foram realizadas no intervalo de $2 \mathrm{~m}$.

Observa-se que nos locais de maior elevação houve maior diferença entre os mapas de curvas e pontos (Figura 4), devido ao fato de que no processo de vetorização de curvas os pontos de máxima cota não são vetorizados e na amostragem por pontos eles foram coletados mostrando, assim, que esta última representa, de maneira mais adequada, a superfície do terreno.

No processo de interpolação do mapa de pontos, devido à obtenção dos pontos de máxima elevação, os pesos dos vizinhos foram melhor distribuídos proporcionando um mapa mais suavizado.

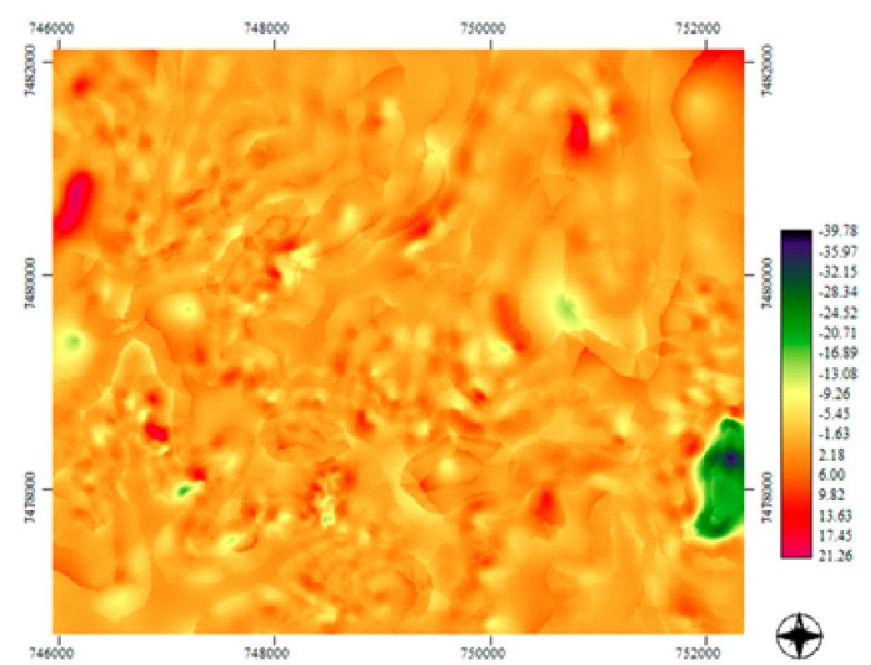

Figura 4. Mapa da diferença entre as metodologias de obtenção dos dados

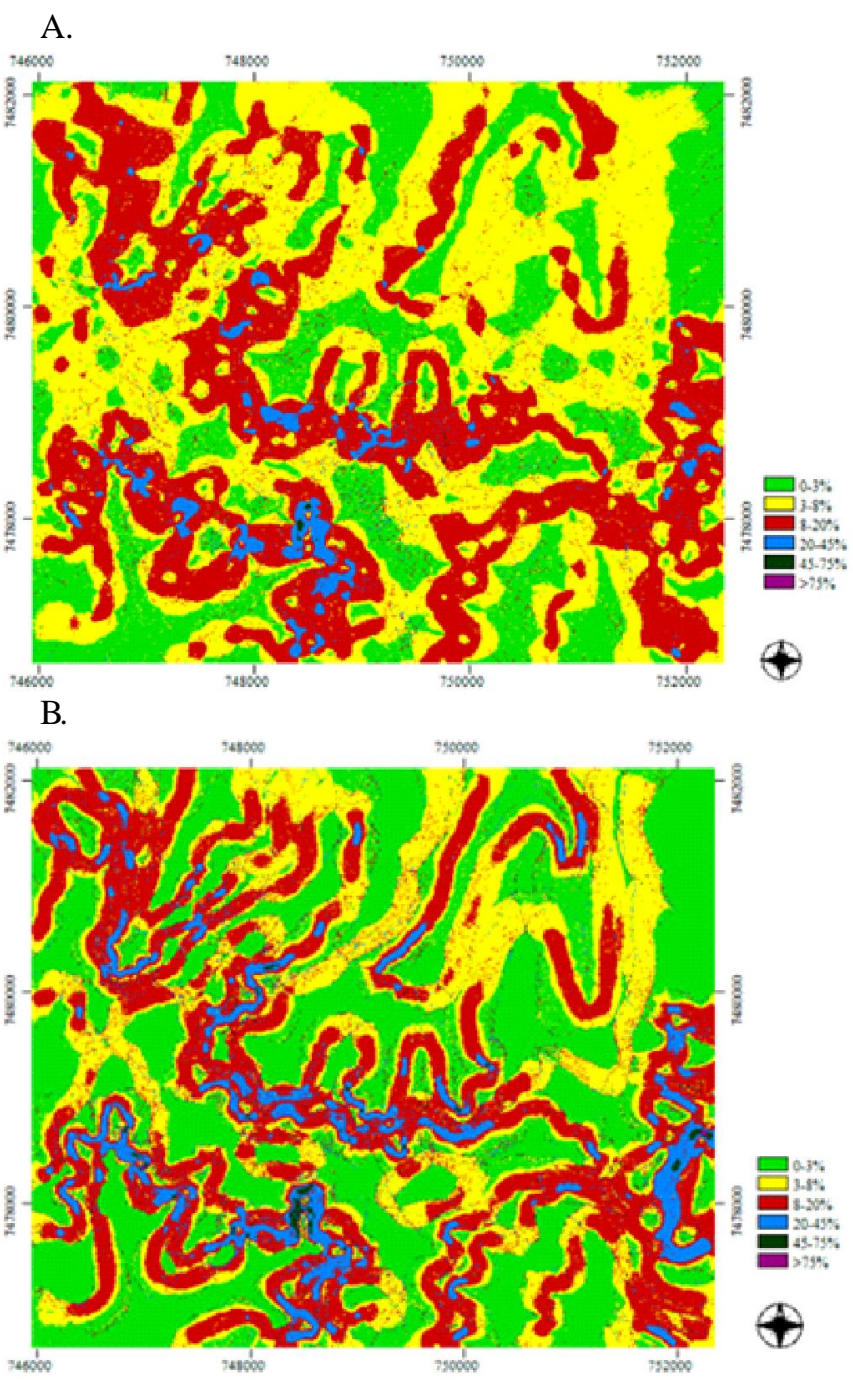

Figura 5. Mapa de declividade dos pontos (A) e de curvas (B)

A diferença nos locais de maiores elevações não influencia na agricultura haja vista que referidas regiões, segundo a legislação, são áreas destinadas à preservação principalmente se o ponto alto está em uma rampa. 
A partir de um MNT é possível gerar um mapa de declividade e este, por sua vez, será utilizado como um dos parâmetros imprescindíveis para determinação das práticas de uso e manejo do solo; assim, quanto mais preciso for o MNT, ou seja, quanto melhor for a amostragem dos pontos de elevação do terreno e sua interpolação, mais representativo será o mapa de declividade.

Embora os mapas de krigagem obtidos tenham distribuição da elevação semelhante, os mapas de declividade demonstraram comportamento distinto entre as classes de relevo (Figura 5).

Na Tabela 1 nota-se que a classe de relevo 0-3\% da krigagem de curvas apresentou maior área (1322,82 ha) e que a krigagem de pontos (849,46 ha); já a área da classe de relevo 3-8\% indicou comportamento inverso, sendo maior para krigagem de pontos (1410,73 ha) e menor para krigagem de curvas (881,21 ha).

Tabela 1. Quantificação das classes de declividade

\begin{tabular}{crr}
\hline \multirow{2}{*}{ Classes de declividade } & Pontos & \multicolumn{1}{c}{ Curvas } \\
\cline { 2 - 3 } & \multicolumn{3}{c}{ (ha) } \\
$0-3 \%$ & 849,46 & 1322,82 \\
$3-8 \%$ & 1410,73 & 881,21 \\
$8-20 \%$ & 1145,09 & 1012,44 \\
$20-45 \%$ & 90,21 & 229,67 \\
$45-75 \%$ & 1,92 & 30,31 \\
$>75 \%$ & 0,14 & 21,09 \\
\hline
\end{tabular}

Nas áreas com declive apropriado para a prática da agricultura, que se encontram no intervalo de 0 a $45 \%$ de declividade, os métodos de amostragem não apresentaram grande diferença em área.

Nas áreas de preservação florestal (declividade acima de $45 \%$ ) ocorreu maior diferença na quantificação de área entre os métodos de amostragem devido à obtenção do ponto de máxima elevação presente na amostragem de pontos, o que proporcionou a suavização do relevo.

Ao realizar a correlação entre os valores dos mapas de krigagem, encontrou-se o $\mathrm{r}^{2}$ de $98 \%$ confirmando a semelhança visual entre os mapas. Na correlação dos mapas de declividade foi encontrado um $\mathrm{r}^{2}$ de $67 \%$ justificado pelo fato do mapa de declividade de pontos ser mais suave que o mapa de declividade de curvas.

\section{ConClusÕES}

1. Os métodos de coleta têm grande diferença no número de pontos.

2. Os variogramas foram ajustados ao modelo exponencial, com alcance aproximado de $1500 \mathrm{~m}$.

3. O método de vetorização apresenta maior grau de dificuldade que quando comparado com a coleta de dados pontuais.

4. O método de coleta de dados pontuais representou adequadamente a superfície do terreno.

\section{AgRAdeCimentos}

Ao GEPAG - Grupo de Pesquisas Agrárias Georreferenciadas e à FCA/UNESP Botucatu, pelo apoio e infraestrutura.

\section{LITERATURA CITADA}

Brocca, L.; Morbidelli, R.; Melone, F.; Moramarco, T. Soil moisture spatial variability in experimental areas of central Italy. Journal of Hydrology, v.333, p.356-373, 2007.

Cichota, R.; Hurtado, A. L. B.; Lier, Q. J. van. Spatio-temporal variability of soil water tension in a tropical soil in Brazil. Geoderma, v.133, p.231-243, 2006.

EMBRAPA - Empresa Brasileira de Pesquisa Agropecuária. Centro Nacional de Pesquisa de Solos. Sistema brasileiro de classificação de solos. 2.ed. Rio de Janeiro: EMBRAPA, 2006. 374p.

Erdogan, S. A comparision of interpolation methods for producing digital elevation models at the field scale. Earth Surface Process and Landforms, v.34, p.366-376, 2009.

Erskine, R. H.; Green, T. R.; Ramirez, J. A.; Macdonald, L. H. Digital elevation accuracy and grid cell size: Effects on estimated terrain attributes. Soil Science Society of America Journal, v.71, p.1371-1380, 2007.

Gonçalves, M. de L. de A. M.; Carvalho, C. A. P. Geração de modelo digital de terreno a partir de mapas digitais 3D: Estudo de caso visando garantir o contexto geomorfológico com redução dos dados amostrais, Boletim de Ciências Geodésicas, v.9, p.105-119, 2003.

Guzzetti, F.; Reichenbach, P.; Cardinali, M.; Galli, M.; Ardizzone, F. Probabilistic landslide hazard assessment at the basin scale.Geomorphology, v.72, p.272-299, 2005.

Hébrard, O.; Voltz, M.; Andrieux, P.; Moussa, R. Spatio-temporal distribution of soil surface moisture in a heterogeneously farmed Mediterranean catchment. Journal of Hydrology, v.329, p.110-121, 2006.

Hengl, T.; Reuter, H. I. (Eds.), Geomorphometry: Concepts, software, applications. Developments in Soil Science, v.33. Amsterdam: Elsevier, 2009, 765p.

Hengl, T.; Bajat, B.; Blagojevic, D.; Reuter, H. I. Geostatistical modeling of topography using auxiliary maps. Computers \& Geosciences, v.34, p.1886-1899, 2008.

IBGE - Instituto Brasileiro de Geografia e Estatística. Cartas planialtimétricas do IBGE, 1972.

Karkee, M.; Steward, B. L.; Aziz, S. A. Improving quality of public domain digital elevation models through data fusion. Biosystems Engineering, v.101, p.293-305, 2008.

Kheir, R. B.; Wilson, J. P.; Deng, Y. X. Use of terrain variables for mapping gully erosion susceptibility in Lebanon. Earth Surface Processes and Landforms, v.32, p.1770-1782, 2007.

Lourenço, R. W. Modelagem geoestatística por geoprocessamento em uma área da baixada santista. Rio Claro: Instituto de Geociências e Ciências Exatas, 2002, 213p. Tese Doutorado

Reuter, H. I.; Kersebaum, K. C.; Wendroth, O. Modeling of solar radiation influenced by topographic shading: evaluation and application for precision farming. Physics and Chemistry of the Earth, v.30, p.139-149,2005.

Robertson, G. P. GS+: Geoestatistics for the environmental sciences - GS+ User's Guide. Plainwell: Gamma Desing Software, 2008. 152p. 
Siska, P. P.; Goovaerts, P.; Hung, I. K.; Bryant, V. M. Predicting ordinary kriging errors caused by surface roughness and dissectivity. Earth Surfaces Process and Landforms, v.30, p.601-612, 2005.

Souza, Z. M.; Martins Filho, M. V.; Marques Júnior, J.; Pereira, G. T. Variabilidade espacial de fatores de erosão em Latossolo Vermelho eutroférrico sob cultivo de cana-de-açúcar. Engenharia Agrícola, v.25, p.105-114, 2005.
Valeriano, M. M. Mapeamento da declividade em microbacias com Sistemas de Informação Geográfica. RevistaBrasileira de EngenhariaAgrícola e Ambiental, v.7, p.303-310, 2003.

Zhu, Y.; Shao, M. Variability and pattern of surface moisture on a small-scale hillslope in Liudaogou catchment on the northern Loess Plateau of China. Geoderma, v. 147, p.185-191, 2008.

Zimback, C. R. L. Análise espacial de atributos químicos de solos para fins de mapeamento da fertilidade. FCA/UNESP: Botucatu, 2001. 114p. Tese Livre-Docência 